\title{
Further experiments on probability learning in goldfish*
}

\author{
WILLIAM T. WOODARD and M. E. BITTERMAN \\ University of Hawaii, Honolulu, Hawaii 96822
}

\begin{abstract}
The prefcrmancs of goldfish in 70:30 and 50:50 visual and spatial problems was studied again under conditions like those employed in recent reversal experiments which provided the first unambiguous evidence of progressive improvement in goldfish. Random matching was found in the visual problems but not in the spatial problems, spatial choices proving rather insensitive to reinforcement ratio. The relation of random matching to the various other outcomes of such experiments is considered.
\end{abstract}

Comparative studies have seemed to show two rather striking differences in the choice behavior of rats and fishes, reversal experiments providing no convincing demonstration in fishes of the progressive improvement found in rats (Behrend \& Bitterman, 1967; Behrend, Domesick, \& Bitterman. 1965: Bitterman, 1969) and probability experiments providing no indication in rats of the random matching found in fishes (Behrend \& Bitterman, 1961; Behrend \& Bitterman, 1966; Bitterman, 1969, 1971). Recent work with a modified training technique makes it clear, however, that progressive improvement does occur in fishes (Engelhardt, Woodard, \& Bitterman, 1973), and the new results have prompted us to look again at probability learning in goldfish using the same technique. If, as others have assumed (Sutherland \& Mackintosh, 1971), the same mechanism underlies "successful" performance both in reversal learning and in probability learning (p. 456), then conditions which yield progressive improvement should tend also to produce maximizing.

\section{METHOD}

Subjects

The Ss were 12 4-in. goldfish, all experimentally naive, divided at random into two equal groups. They were maintained in individual 2 -gal tanks at $21^{\circ} \mathrm{C}$ on a 24 -h feeding schedule.

\section{Apparatus}

The apparatus used here and in our most recent work on reversal learning (Engelhardt et al. 1973) is a modification of an earlier apparatus which is illustrated elsewhere (Bitterman. 1965). It consists of a black Plexiglas enclosure to which the animal is carried in its individual living tank. At one end of the enclosure are two circular targets of diffusing Plexiglas on which the stimuli to be discriminated (red and green lights) are projected, and the animal chooses between them by striking one

*This research was supported by Grants MH23294 (formerly 17736) and NS06890 from the United States Public Health Service. The assistance of Fred Engelhardt in collecting the data is acknowledged with thanks. Requests for reprints should be addressed to the Laboratory of Sensory Sciences, University of Hawaii, 1993 East-West Road, Honolulu. Hawaii 96822. of the targets. In the earlier apparatus, correct choice was rewarded with a Tubifex worm discharged by a feeder at the other end of the enclosure. In the modified apparatus, reward is presented at the locus of the correct response, each target having a plastic nipple at its center through which liquid food (Biorell and cornstarch cooked with water) can be delivered (Holmes \& Bitterman, 1969). Another modification is the addition of a center key $(\mathrm{CK})$ at the end of the enclosure opposite the targets. The $\mathrm{CK}$ and the targets are mounted-as in the earlier apparatus-. on light rods clamped into the needle holders of crystal phonocartridges (Astatic 16), and contacts are detected electrically with a circuit described elsewhere (Holmes \& Bitterman, 1969). All experimental events are programmed automatically.

\section{Procedure}

The animals were trained in daily sessions of 40 trials. Each trial began after an intertrial interval of $6 \mathrm{sec}$ in darkness with the illumination of the CK by a white lamp. Response to the CK turned off the lamp and caused the pair of targets at the opposite end of the enclosure to be illuminated by red or green lamps. Correct choice-i.e., choice of the target scheduled as positive on any given trial -initiated a 6 -sec reinforcement cycle during which the discriminanda were turned off and the chosen target was illuminated by a white lamp. An initial drop of food was delivered through the nipple of the chosen target at the start of the cycle, and each further contact with the target during the cycle produced an additional drop of food. Incorrect choice produced a 10 -sec time-out in darkness, after which the $\mathrm{CK}$ was illuminated once more. Response to the $\mathrm{CK}$ turned it off and illuminated (with the appropriate color) only the correct target. response to which initiated a reinforcement cycle (the so-called guidance procedure).

In the first stage of the experiment, both groups-visual and spatial-were trained for 23 sessions on 100:0 problems. For the visual group. one color was consistently reinforced and the other never red for three of the animals and green for the others. For the spatial group. one position was consistently reinforced and the other never - left for three of the animals and right for the others. For both groups, one target was red and the other green on each trial, the positions of the two colors varying from trial to trial in accordance with Gellermann orders. Color. then, was the relevant dimension for the visual group and an irrelevant one for the spatial group. while position was relevant for the spatial group and irrelevant for the visual group.

In the second stage of the experiment. the visual animals were trained for 17 sessions and the spatial animals for 16 sessions on $70: 30$ problems. The consistently reinforced stimulus of the first stage of the experiment (the majority stimulus) now was reinforced on only $70 \%$ of trials. while the consistently unreinforced stimulus of the first stage (the minority stimulus) 


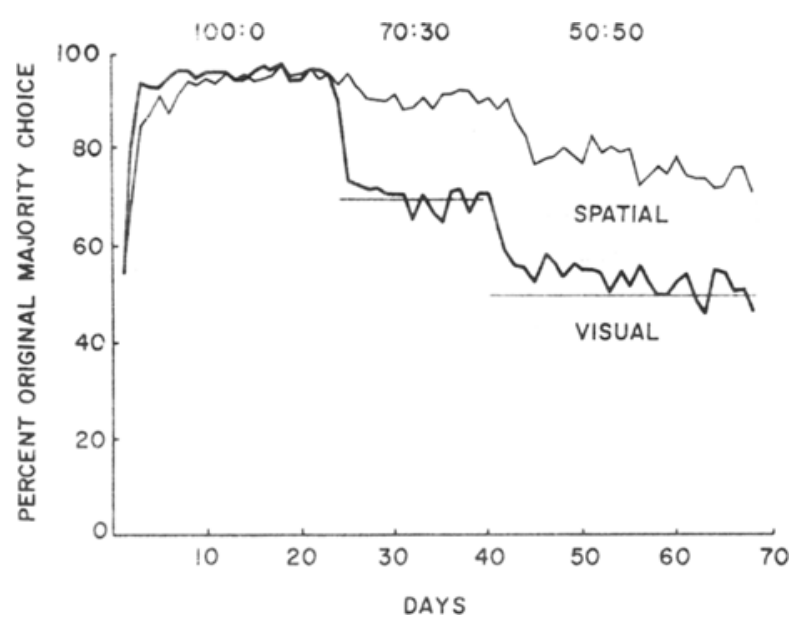

Fig. 1. The performance of the two groups in each of the three stages of training plotted in terms of mean percent choice of the stimulus consistently reinforced in the first stage.

now was reinforced on the remaining $30 \%$ of trials. The schedules of reinforcement were constructed with the restriction that there be at least one minority reinforcement in each block of five trials and no more than two such reinforcements in succession. For the visual animals, each color was reinforced as often on the left as on the right. For the spatial animals, each color appeared as often in the reinforced as in the unreinforced position

In the thitr stage of the experiment, both groups were trained on 50:50 problems for 28 or 29 sessions. For the visual group, color continued to vary, one key being red and the other green on each trial, their spatial arrangement being determined again by Gellermann orders, but neither color nor position was differentially reinforced. For the spatial group, the two keys always were the same color--red for three of the animals and green for the others. The schedules of reinforcement, both as to color and position in the visual group and as to position in the spatial group, were constructed on the basis of Gellermann's rules.

\section{RESULTS}

In Fig. 1, the performance of the two groups is plotted in terms of the percentage of trials in each session on which the consistently reinforced stimulus of the first stage (the original majority stimulus) was chosen. Both groups rapidly acquired a strong preference for that stimulus in the 100:0 training. In the subsequent 70:30 and 50:50 training, the visual animals tended to match, their choice ratios closely approximating the reinforcement ratios, but there was no suggestion of matching in the performance of the spatial animals. The preference established in the 100:0 spatial training was reduced to some extent as the schedule of reinforcement changed to $70: 30$ and then to $50: 50$, but the choice ratios were substantially greater than the reinforcement ratios.

The 70:30 and 50:50 performance of individual animals 't the visual group is plotted in the left-hand portion of Fig. 2. Choices both in the relevant dimension (original majority color irrespective of position) and in

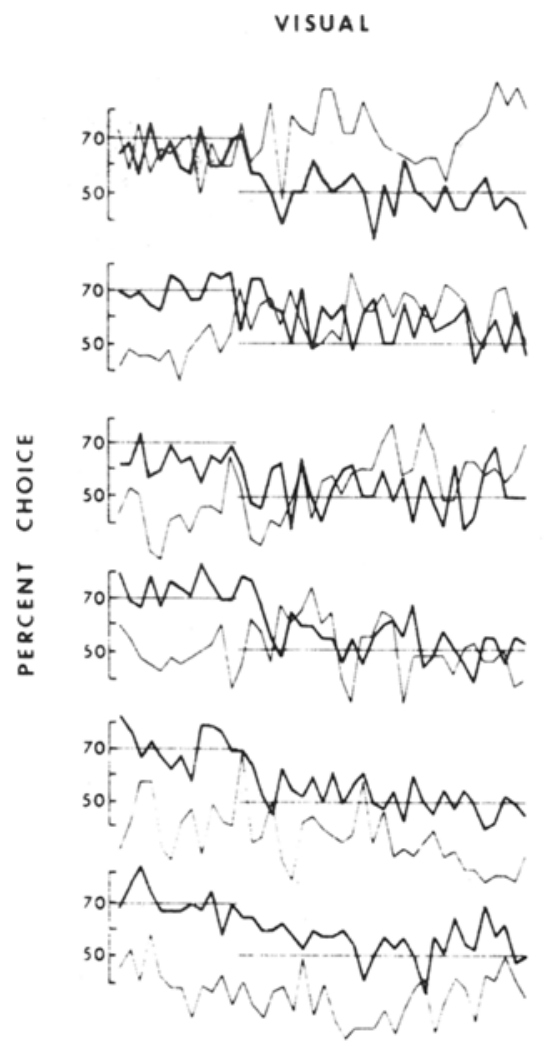

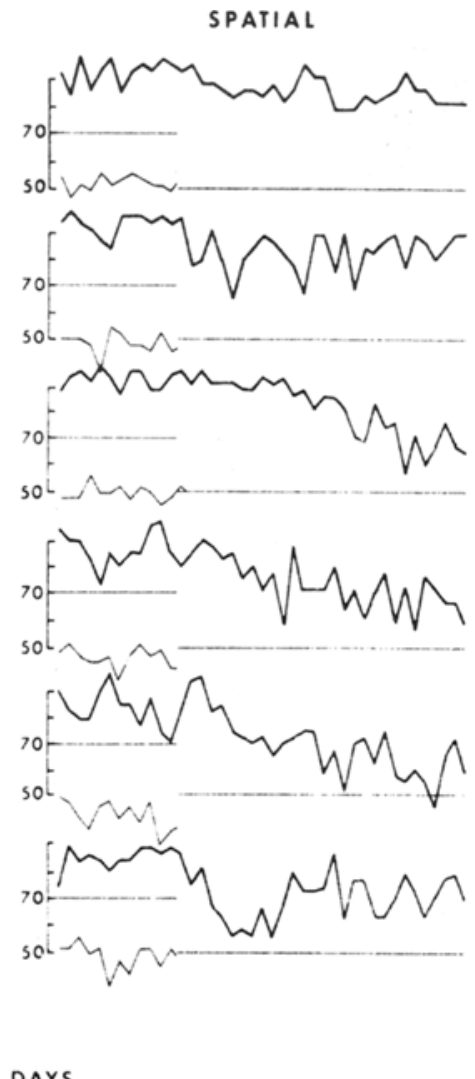

Fig. 2. The performance of individual animals, visual and spatial, in 70:30 and 50:50 training sessions. The heavier lines show choices in the relevant dimension (color for the visual animals and position for the spatial animals), while the lighter lines show choices in the alternative dimension. 
the irrelevant dimension (left-hand target irrespective of color) are shown. Visual matching is evident in the data for each animal, i.e., percent choice of the original majority color comes closely to approximate 70 in the $70: 30$ problem and 50 in the 50:50 problem. Furthermore, the visual matching can in no sense be regarded as an artifact of spatial bias, as sometimes, for example, it has been found to be in work with rats, when equal response to two visual alternatives in a 50:50 problem occurs as the inevitable consequence of consistent choice of one or the other spatial alternative (Bitterman, 1971). Spatial preferences vary considerably both within and between animals, but there seems to be no relation between visual and spatial choices.

Computer analyses were made of sequential dependencies in the data for each visual animal in the last 10 sessions of $70: 30$ training and the last 10 sessions of 50:50 training. Visual choice and spatial choice on Trial $\mathrm{N}$ were examined in relation to visual choice, spatial choice, and correctness of choice on Trial N - I. Of 1802 by 2 chi-square tests ( 30 tests for each of six animals) in the 70:30 condition. only 10 were significant $(p<.05)$; of a like number of tests in the 50:50 condition, only 12 were significant. These results suggest that there were no substantial dependencies in the data. It is interesting to note that, of all the strategies investigated, only win-stay with respect to color would tend to produce matching in the $70: 30$ problem, yet there was no evidence of such a strategy in the data. One of the six visual aninals (the third from the top in Fig. 2) did show a significant relation between color chosen on Trial $\mathrm{N}$ and color rewarded on Trial $\mathrm{N}-1$, but the relationship was negative (win-shift), which may account for the fact that percent majority choice in this animal was somewhat below 70 . Another interesting analysis has to do with the relation between response to color and response to position on Trial N. Of 12 analyses (one for each animal in 70:30 and one for each animal in 50:50), only 1 yielded a significant chi square $(p<.05)$, which is about what might be expected by chance. It may be concluded that choice of color and choice of position were independent.

The 70:30 and 50:50 performance of individual animals in the spatial group is plotted in the right-hand portion of Fig. 2. Choices both in the relevant dimension (original majority position irrespective of color) and in the irrelevant dimension (red target irrespective of position) are shown for the 70:30 problem. For the 50:50 problem, only spatial choices are shown, since both targets were the same color on each trial. Nothing like spatial matching is evident in the results for any of the animals, percent choice of the original majority color substantially exceeding the reinforcement ratio in each case. The 70:30 data show no substantial bias with respect to choice of color, which, of course, was nondifferentially reinforced. Here again, then, color choices are sensitive to reinforcement ratio. while spatial choices are not.

\section{DISCUSSION}

When goldfish are trained under conditions which produce progressive improvement in reversal as compared with conditions which do not, visual probability matching persists while spatial prubability matching does not. These results are in accord with previous results for pigeons which show that matching is sensitive to such training variables as the kind of correction procedure employed and the presence or absence of a CK (Graf, Bullock, \& Bitterman, 1964); with unlimited correction, visual matching occurs in pigeons whether or not a $\mathrm{CK}$ is used, but with guidance (a procedure which controls the distribution of reinforcement without permitting repetitive errors). visual matching occurs only when a $\mathrm{CK}$ is not used. The failure of goldfish to show spatial matching under circumstances in which they show visual matching also is in accord with previous results for pigeons (Bullock \& Bitterman, 1962; Graf et al. 1964), as well as for painted turtles (Kirk \& Bitterman, 1965) and for decorticated rats (Gonzalez, Roberts, \& Bitterman, 1964), in none of which spatial matching has been found.

However restricted the range of conditions in which matching can be demonstrated, the fact remains that it does occur in a variety of submammalian vertebrates, while it never has been found in intact mammals. Correspondences between choice ratio and reinforcement ratio which have appeared in mammals have proved. when examined closely, to be artifacts either of grouping or of systematic response in individual Ss to other features of the training situation. such as, for example. locus of reward on the preceding trial. The visual matching displayed in Fig. 2 clearly is an individual phenomenon, and analysis of sequential dependencies demonstrates that it is random not. of course, in the sense that the animals are unbiased in their choices of stimuli other than color, but in the sense that the correspondence is not an artifact or secondary consequence of systematic response to other stimuli (Behrend \& Bitterman, 1966). The conclusion seems warranted that the determinants of random matching are in some sense unique, different in kind from those which produce either nonrandom (strategic) matching or maximizing.

Sutherland and Mackintosh (1971) have proposed that all of these behaviors as they occur in a varicty of animals, and the data on reversal learning as well. can be derived from attentional theory on the assumption that animals differ quantitatively in their ability to maintain attention to relevant stimuli in the face of inconsistent reinforcement. Matching they hold to be not a distinctive phenomenon, but simply one point on a continuum of many possible outcomes dictated by an array of parameters, both subjective and situational. If that were true, however, it would be difficult to understand why such close correspondence between choice ratio and reinforcement ratio should be found in 
several different species tested under widely varying circumstances. In any case, two aspects of the present results seem directly to contradict the attentional interpretation: One is that goldfish continue to show visual matching under conditions which not only facilitate acquisition of 100:0 training, but which also yield progressive improvement in reversal and which therefore must be assumed by Sutherland and Mackintosh to strengthen attention. A second is that the spatial biases shown by the visual animals were no greater when they chose the minority color than when they chose the majority color. If minority choice is due to shift of attention from the relevant to an irrelevant dimension. then any preference in an irrelevant dimension should be more evident on those trials. It is not enough. as Mackintosh, Lord, and Little (1971) have done, merely to look at minority choices alone; the theory requires a disproportionality, of which no evidence was found here.

\section{REFERENCES}

Bitterman. M. E. The evolution of intelligence. Scientific American, 1965, 212, 92-100.

Bitterman, M. E. Habit-reversal and probability learning: Rats, birds, and fish. In R. M. Gilbert and N. S. Sutherland (Eds.), Animal discrimination learning. London and New York: Academic Press, 1969.

Bitterman. M. E. Visual probability learning in the rat.
Psychonomic Science, 1971. 22, 191-192.

Behrend. E. R., \& Bitterman, M. E. Probability-matching in the fish. American Journal of Psy'chology, 1961. 74, 542-551.

Behrend. E. R., \& Bitterman, M. E. Probability-matching in the goldfish. Psychonomic Science. 1966, 6. 327-328.

Behrend. E. R., \& Bitterman. M. E. Further experiments on habit reversal in the fish. Psychonomic Science. 1967. 8. 363-364.

Behrend. E. R.. Domesick, V. B., \& Bitterman, M. E. Habit reversal in the fish. Journal of Comparative \& Physiological Psychology, 1965, 60, 407-411.

Bullock. D. H., \& Bitterman. M. E. Probability-matching in the pigeon. American Journal of Psychology, 1962, 75, 634-639.

Engelhardt. F.. Woodard. W. T., \& Bitterman, M. E. Discrimination reversal in the goldfish as a function of training conditions. Journal of Comparative \& Physiological Psychology, 1973, in press.

Gonzalez, R. C., Roberts. W. A., \& Bitterman. M. E. Learning in adult rats with extensive cortical lesions made in infancy. American Journal of Psychology, 1964, 77, 547-562.

Graf, V., Bullock, D. H., \& Bitterman, M. E. Further experiments on probability-matching in the pigeon. Journal of the Experimental A nalysis of Behavior, 1964, 7, 151-157.

Holmes, N. K., \& Bitterman, M. E. Measurement of consummatory behavior in the fish. Journal of the Experimental Analysis of Behavior, 1969, 12, 39-41.

Kirk, K. L., \& Bitterman. M. E. Probability-learning by the turtle. Science, 1965, 148. 1484-1485.

Mackintosh, N. J., Lord, J.. \& Little, L. Visual and spatial probability learning in pigeons and goldfish. Psychonomic Science, 1971, 24, 221-223.

Sutherland, N. S., \& Mackintosh, N. J. Mechanisms of animal discrimination learning. London and New York: Academic Press, 1971.

(Received for publication September 28, 1972; accepted October 9.1972.) 